\title{
Effect of Grain Boundary Structure on Defect Absorption and Denuded Zone Formation in Irradiated Nanocrystalline Iron
}

\author{
O. El-Atwani ${ }^{1}$, k. Hattar ${ }^{2}$, G. Vetterick ${ }^{1}$, M.L. Taheri ${ }^{1}$ \\ 1. Drexel University, Department of Materials Science \& Engineering, Philadelphia, PA USA. \\ 2. Department of Radiation Solid Interactions, Sandia National Laboratories, NM, United States
}

The performance of nuclear materials in extreme environments poses important fundamental questions about the behavior of condensed matter under far-from-equilibrium conditions.[1] Nuclear materials are exposed to high heat flux and irradiation that alter their microstructure, mechanical properties, and performance.[2] To mitigate possible damage, the use of UltraFine (UF) and NanoCrystalline (NC) metals has been proposed, due to their high grain boundary densities that thus act as high defect and particle sinks, [3] and improve mechanical properties (strength and ductility). [4] While recent research has focused on the effect of grain boundary character [5] on the radiation tolerance, fundamental questions about the role of non-equilibrium grain boundaries in defect absorption in comparison with equilibrium grain boundaries have yet to be answered. Understanding the effect of the non-equilibrium boundary local strain in irregular defect absorption and denuded zone formation could be crucial in engineering polycrystalline materials of higher radiation tolerance that can sustain the severe environments of future nuclear reactor conditions. In this work, in-situ irradiation was performed in a transmission electron microscope (TEM) on freestanding nanocrystalline Fe samples of less than 100 $\mathrm{nm}$ grain size. To form the nanocrystalline samples, Fe films was sputter-deposited on $\mathrm{NaCl}$ substrates [6]. TEM micrographs of the samples after annealing showed both equilibrium and non-equilibrium (high local strain manifested by strong extinction bands) boundaries adjacent to each other (Figure.1), thus enabling the comparison between both boundary types. The in-situ TEM irradiation experiments were performed using the i3TEM facility in the Department of Radiation Solid Interactions at Sandia National Laboratories. Crystallographic orientation microscopy (ACOM) was also performed via NanoMEGAS ASTAR precession diffraction (eg. Figure.2). Effect of defect absorption on grain boundary structure is studied through the in-situ irradiation/ACOM experiments. The findings provide fundamental aspects to be considered in engineering high radiation tolerant nanomaterials. 
References:

[1] J. Hemminger et al., "Directing Matter and Energy: Five Challenges for Science and the Imagination." Department of Energy's Office of Science (http://science. energy. gov/ /media/bes/pdf/reports/files/gc_rpt. pdf) (2007).

[2] A. Makhankov et al., J Nucl Mater, 290 (2001) 1117

[3] T.D. Shen et al., Appl Phys Lett, 90 (2007) 263115

[4] Y. Wang et al., Nature, 419 (2002) 912

[5] C.M. Barr et al., Acta Materialia, 67 (2014) 145

[6] G. Vetterick et al., J. Appl. Phys, 116 (2014) 233503

[7] M.L.Taheri, G. Vetterick, and O. El-Atwani gratefully acknowledge funding from the United States Department of Energy, Basic Energy Sciences under the Early Career program through contract DESC0008274. K. Hattar acknowledges the Division of Materials Science and Engineering, Office of Basic Energy Sciences, U.S. Department of Energy. Sandia National Laboratories is a multi-program laboratory managed and operated by Sandia Corporation, a wholly owned subsidiary of Lockheed Martin Corporation, for the U.S. Department of Energy's National Nuclear Security Administration under contract DE-AC04-94AL85000.

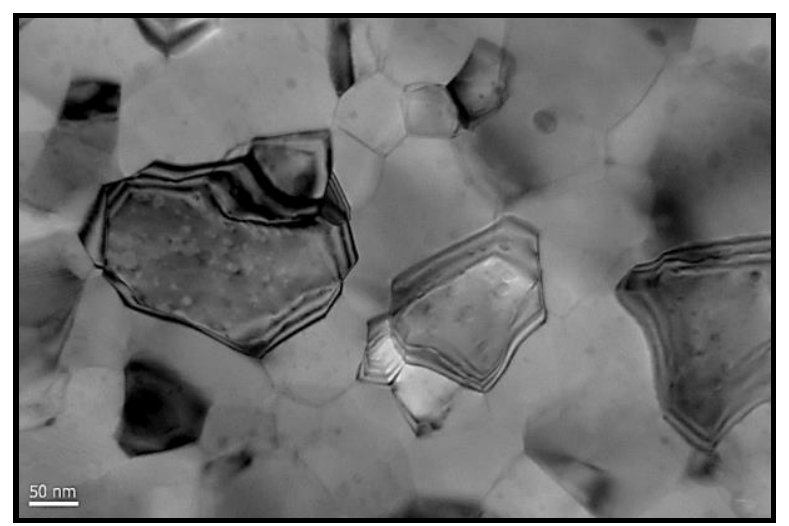

Figure 1. Bright field TEM image of nanocrystalline iron film annealed at $500{ }^{\circ} \mathrm{C}$.

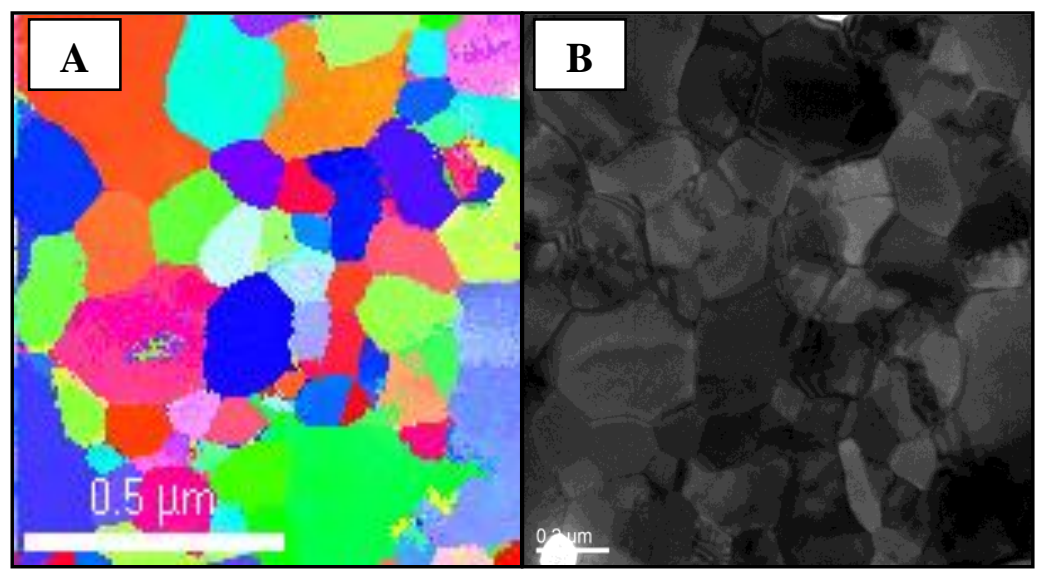

Figure 2. Microstructure of an annealed iron film. (A) Crystallographic orientation map and (B) the corresponding TEM bright field image. 